\title{
Le voyage d'Italie et la formation des élite françaises
}

JEAN

BALSAMO

Summary: During the sixteenth century, at least until 1559, Italy was a centre for political, diplomatic, and cultural activity for the French elite, who undertook studies and training in the Peninsula. Lawyers and magistrates in the making eagerly enrolled in the universities of Pavia, Ferrara, and especially Padua, where some of them joined with other scholars in constituting the first Literary Republic. Gentlemen who were aiming at a military career or wished to succeed at the Court went to the Academies of Naples, Bologna or Padua, where they learned fencing and riding. During the age of Henri IV, attitudes towards this double education, scholarly and aristocratic, changed thoroughly, partly for economic reasons, but also because of a general anti-Italian polemic which opposed the model of Italian culture and social structure to a national model. The French did continue to visit Italy during the entire early modern period, but latterly they went to "see," not to learn.

E n conclusion du journal de son voyage en Terre sainte et en Italie, Nicolas Bénard, un jeune bourgeois parisien, faisait le catalogue des agréments qu'offrait la Péninsule au début du XVII ${ }^{e}$ siècle. Il considérait que l'Italie était un pays admirable, dans lequel le pèlerin trouverait tout ce qu'il pouvait souhaiter pour satisfaire sa dévotion et ses curiosités. Mais à la leçon qu'il tirait de sa propre expérience, Bénard ajoutait que

les curieux et désireux de sciences et arts libéraux ou mécaniques y apprendront tout ce qui leur restera pour la perfection de leur vacation ${ }^{1}$.

Il traçait ainsi le tableau d'une Italie remplie de « sçavans et doctes personnages en toutes sortes de sciences et arts », comme une sorte de Parnasse, ou mieux, comme un conservatoire universel dont il fallait se hâter de 
recueillir l'enseignement. De fait, dans ce qui peut nous apparaître comme un banal lieu commun, Bénard avait l'intuition à la fois d'une transformation en profondeur du rôle pédagogique de l'Italie qui avait été pendant cent cinquante ans le lieu de tous les savoirs, ainsi que du rapport que les Français pouvaient entretenir avec elle. Vers 1620 , en effet, les peintres commençaient à venir se former à Rome ou à Venise ${ }^{2}$. Au même moment, la noblesse, rompant une tradition vieille de plusieurs générations et une ancienne connivence, désertait sinon l'Italie, qui gardait pour elle son pouvoir d'attraction, fût-ce comme la proie de ses ambitions militaires, du moins les universités et les lieux où elle avait jusqu'alors cherché le couronnement de sa formation.

\section{La tradition des études italiennes}

Dans une lettre peu connue, Jacques Amyot traçait un programme d'éducation et un plan de carrière à l'usage du fils de Jean de Saint-Sulpice, que la faveur du souverain destinait au siège épiscopal d'Agen. Le jeune homme devait faire le voyage de Rome ; il y formerait ainsi son caractère et sa prudence, par «l'expérience d'avoir vu à l'œil les mœurs, pays et façons de faire des étrangers », il y approfondirait sa connaissance de l'antiquité profane et de l'antiquité chrétienne, il y poursuivrait des études de théologie et de philosophie sacrée, au sein de "la meilleure école de religion qui saurait être », le Collegio romano, en suivant les cours des Jésuites et des Hiéronymites ${ }^{3}$. Ce projet correspondait à la première des cinq professions nobles telles qu'allait les définir Antoine de Laval, « [de] Théologie, des armes, de jurisprudence, de secrétaire ou des finances $»^{4}$, dans un traité destiné à relever la France ruinée par les guerres civiles, en impliquant la noblesse dans de nouveaux domaines de compétence. Les trois premières «professions » toutefois restaient les plus prestigieuses, et les jeunes gens, que leur famille destinait aux carrières de la robe ou de l'épée, après une formation initiale dans leur propre pays, allaient souvent se perfectionner dans ces disciplines en Italie.

Les juristes français se formaient généralement à Orléans ou à Bourges, puisque le droit n'était pas enseigné à Paris. Mais ils étaient aussi attirés par les universités italiennes, même si celles-ci ne bénéficiaient d'aucune exclusivité 5 . Ils fréquentaient plus volontiers Pavie, où l'on recense plus de quatre cents d'entre eux, aux $\mathrm{XV}^{\mathrm{e}}$ et $\mathrm{XVI}^{\mathrm{e}}$ siècles ${ }^{6}$, Ferrare, qui attira plus de trois cents Français jusqu'en 1559, attirés par la présence de la duchesse Renée ${ }^{7}$, Bologne, ainsi que le relate le Journal de Nicolas Audebert ${ }^{8}$, et surtout Padoue $^{9}$. Le prestige de certains maîtres, le fait que certains d'entre eux avaient aussi, tel Alciat, enseigné en France, contribuaient à cet attrait. 
L'intérêt pour les études de droit tenait certes aux origines sociales des étudiants, mais aussi au fait que le droit n'était pas enfermé dans des définitions étroitement nationales. En outre, l'Italie représentait, jusqu'en 1559 du moins, un champ d'action administratif et politique pour les élites françaises, qui trouvaient en Piémont des possibilités de carrière, où ils faisaient jouer, à l'exemple de leurs aînés, les réseaux de solidarités ainsi constitués. Le rôle des anciens de Padoue dans la haute administration royale et les parlements sous Henri II est bien connu ${ }^{10}$. La célèbre université de Vénétie n'avait cessé, durant tout le siècle, de recevoir de forts contingents étrangers, parmi lesquels, à côté des Allemands et des Anglais ${ }^{11}$, les Français, répartis entre Bourguignons et Provençaux, étaient nombreux : on en dénombrait cinquante en 1539, quarante-huit en 1564, soixante-quatre en 1587. Parmi tant d'autres qui y firent leurs études, à l'époque de "l'Italie françoise », Michel de L'Hospital, Pierre de Mondoré ou Arnaud du Ferrier ; ce dernier y prit ses grades et fut même chargé d'un enseignement, avant de devenir ambassadeur à Venise ; dans le dernier quart du siècle ; on notera en particulier François de Joyeuse, futur cardinal, qui y séjourna de 1573 à 1581, Claude Expilly, Pierre Virey, qui laissa de son séjour d'intéressants Vers itinéraires ${ }^{12}$, François de Sales et surtout Peiresc. Ce dernier avait su convaincre sa famille de l'envoyer achever son droit en Italie, qu'il désirait depuis longtemps visiter, à la suggestion d'un ami qui lui avait fait comprendre qu'il pourrait satisfaire d'autres curiosités savantes : «interponere juris studio rerum antiquarum pervestigationes $\gg 13$.

Les étudiants suivaient en effet d'autres cours et s'intéressaient à d'autres disciplines que le droit, comme des compléments, selon leurs goûts et le prestige des maîtres. Virey avait étudié un peu de médecine en auditeur libre des cours de Mercuriali, Peiresc suivit les cours d'anatomie de Girolamo Aquapendente, et il s'initia aux mathématiques. Mais dans l'ensemble, peu de médecins français allèrent se former en Italie. Les étudiants français renonçaient fréquemment à passer leur diplôme en Italie. Nicolas Audebert, qui avait étudié pendant trois ans à Bologne et qui y avait assumé les fonctions de recteur étranger, revint passer sa licence à Bourges, sous Cujas. Peiresc lui-même, fut reçu docteur à Montpellier. Ces deux exemples illustrent sans doute des choix individuels, mais confirment aussi des stratégies universitaires et de carrière. Ils semblent aussi indiquer les limites de la formation reconnue, offerte par un séjour en Italie. Dans une lettre à son oncle, Peiresc jugeait en termes sévères l'enseignement qu'il avait reçu :

Car enfin tout ce qu'avons apprins à Padoue, et toutes les leçons qu'y avons ouy, ne nous ont apporté qu'une cognoissance du Droict fort confuse, avec un mélange de diverses choses tant utiles qu'inutiles ${ }^{14}$. 
Au début du XVII ${ }^{e}$ siècle, pour diverses raisons, les universités italiennes connurent une désaffection générale de la part des Français : ceux-ci ne furent plus que seize à faire leurs études à Padoue, pour toute la période de 1622 à 1641. Les raisons économiques devaient primer, ainsi que les nouvelles possibilités d'études en France même ; les raisons religieuses, généralement invoquées semblent moins probantes, les protestants français allaient depuis plus d'un demi-siècle en Allemagne et en Suisse ou dans les Pays-Bas. Cette désaffection tenait sans doute à ce qui était considéré comme un déclin des études en Italie, et le déplacement vers le nord du centre de gravité de l'Europe savante.

\section{La conversation des savants}

Au cours du XVIe siècle, les plus lucides ou les plus désabusés des observateurs de la vie savante portaient sur l'activité intellectuelle de l'Italie un jugement sévère : celle-ci n'offrait plus les meilleurs maîtres dans les études humanistes ; elle était dépossédée de son héritage antique au profit de la France et plus généralement des érudits du nord. Ces jugements constituaient en fait les éléments topiques d'un discours hostile à l'Italie et qui s'était développé depuis Érasme, complaisamment repris par les savants gallicans et les propagandistes d'une translatio studii au profit de la couronne de France. Ils avaient une portée polémique qui ne rendait compte ni de l'extraordinaire activité artistique dont l'Italie (le " grand atelier », selon l'expression d'André Chastel) était le théâtre, ni des développements brillants de la littérature en langue vulgaire. L'Italie tridentine, sous des papes lettrés, ne cessera de jouer au XVII siècle encore ce rôle de Parnasse européen ${ }^{15}$. Il n'y avait pas de contradiction toutefois entre cette brillante vie « culturelle » et le déclin réel, mais difficile à estimer des universités. Jusqu'au tournant du siècle, comme en porte témoignage l'éloge liminaire de Bénard, les Français souscrivaient sans réticence à la réputation savante de l'Italie, et ils cherchaient à rencontrer les grands personnages qui l'illustraient.

La rencontre des érudits italiens, la visite au grand savant, qui pouvait donner à l'expérience italienne toute sa prégnance, et confirmer au voyageur une nouvelle autorité, constituait un «lieu » du récit de voyage, confirmé par la répétition des mêmes usages et des mêmes noms. Jacques-Auguste de Thou ne fit pas, stricto sensu, un séjour universitaire en Italie, mais son voyage à la suite de l'ambassadeur Paul de Foix, était bien un voyage d'études ${ }^{16}$. Avant son départ, en compagnie de son protecteur, il avait étudié les commentaires d'Alessandro Piccolomini sur la Physique d'Aristote, et lors de son passage à Sienne, il alla rencontrer le vieil érudit, qui bénéficiait 
alors d'une brillante réputation auprès des lettrés français ${ }^{17}$. Lors d'une étape à Padoue, un même intérêt lui fit rendre visite à Mercuriali, à Patrizi, à Nifo le jeune, dont il donne les noms dans ses Mémoires. À Rome, de Thou fut introduit par Arnaud d'Ossat et par Muret auprès de Paul Manuce et de Fulvio Orsini, qui lui ouvrit ses collections et sa bibliothèque ; à Naples, il rencontra Della Porta ; à Bologne enfin, il avait fréquenté Sigonio, qui le força « pour ne pas se priver de sa conversation, de se servir du mieux qu'il pût de l'Italien ». De la même manière, les études de Nicolas Audebert à Bologne trouvèrent leur prolongement, au cours de plusieurs voyages à travers la péninsule, dans un même réseau de rencontres érudites, rendues possibles grâce aux recommandations de Claude Dupuy et de Pinelli. Le jeune érudit relate en particulier sa rencontre et ses longues conversations avec Piero Vettori ${ }^{18}$.

Si l'on en croit le récit tardif qu'en donne Gassendi, le séjour italien de Peiresc fut même tout entier organisé en vue de ces rencontres savantes. Déçu de l'enseignement qu'il recevait dans les universités, Peiresc alla chercher ailleurs les lieux où se cultivait le savoir, et recevoir d'autres marques d'approbation que des diplômes. Introduit auprès de Pinelli, il fut reconnu comme un disciple par le savant padouan, comme l'avait été Claude Expilly vingt ans avant lui ${ }^{19}$. Peiresc put en outre se faire ouvrir tous les cabinets de curiosité et être admis dans les cercles lettrés les plus fermés de la péninsule, "quia jam Pinelli aliorumque celebre nomen fecerant», rencontrant Baronio, Bellarmin, Fulvio Orsini et tant d'autres. Ces relations, entretenues par des lettres, des services, des envois de livres et d'informations, d'autres recommandations, se précisaient en une culta amicitia, cette forme de sociabilité si active dans ses manifestations, sur laquelle allait reposer, pour près de quarante ans, une République des Lettres gouvernée par Peiresc, qui sut donner une tout autre ampleur au réseau tissé par Pinelli. Celui-ci, en mourant, aurait désigné son jeune protégé comme le continuateur de sa mission, «quod videretur Peireskio quasi lampada tradidisse ». Ainsi interprété par Gassendi, le voyage d'études de Peiresc devenait un véritable sacre érudit. Reconnu et couronné par les savants d'Italie et d'Europe, Peiresc assumait la succession de Pinelli, mais il symbolisait surtout, aux yeux des Français, la translatio studii ${ }^{20}$.

Les rencontres avec les lettrés et les savants italiens entraient dans les marges du voyage d'études au sens strict. Sous d'autres formes et avec d'autres personnages, elles offraient l'occasion d'un autre enseignement, le plus prestigieux, destiné aux futurs magistrats au service de la monarchie. Rome était la véritable capitale politique de l'Europe, la Curie apparaissait comme une des administrations les plus complexes et les plus efficaces. Dans sa lettre à Jean de Saint-Sulpice, Amyot rappelait que l'Italie, par la 
fréquentation, la conversation des prélats et des diplomates, était le lieu du monde le plus idoine «à entendre les affaires », afin de pouvoir un jour « les manier dextrement pour le service de l'Église et des Princes ». Le jeune de Thou, par sa situation privilégiée auprès de Paul de Foix, sut ainsi se faire introduire dans les arcanes d'une cour où se traitait alors le sort du monde ; il reçut l'enseignement du cardinal Santacroce, après avoir été initié à la politique florentine par Vasari.

\section{La culture de la noblesse}

Tous les jeunes Français ne venaient pas en Italie pour rencontrer des savants ou suivre des cours. D'autres rencontres et d'autres formes d'éducation répondaient aux besoins de la noblesse, et au sommet des hiérarchies aristocratiques, certains princes français recevaient aussi une expérience de cour à l'italienne, alors qu'au même moment des seigneurs italiens se formaient à la cour de France. En 1584, le prince de Joinville, fils du duc de Guise et petit-fils d'Anne d'Este, alors âgé de treize ans, vint passer deux ans à la cour de Ferrare $^{21}$. Le Tasse célébra dans plusieurs poèmes ce descendant du héros de sa Gerusalemme liberata ${ }^{22}$.

Les simples gentilshommes qui se destinaient à la carrière des armes ou qui désiraient se faire un chemin à la cour, fréquentaient les académies de Naples, de Bologne ou de Padoue, où ils faisaient leurs « exercices ». Le jeune Charles de Villeroy, formé en l'Italie par son précepteur Lourdereau, et par Belleforest, qui lui avait dédié sa traduction d'un des textes majeurs de la civilité aristocratique, la Civile Conversatione de Stefano Guazzo, vint à Padoue, à l'âge de 17 ans. Dans son journal de voyage, resté manuscrit, il évoquait avec précision la formation qu'il venait suivre :

J'ay recommencé de tirer des armes le 1er jour de Juin 1584. J'ay recommencé à danser le 3. Juin 1584. J'ay commencé à voir un maistre Italien le 7. Juin 1584. J'ay commencé les Mathématiques le 10. juin $1584^{23}$.

Villamont, auteur d'un fameux récit de voyage en Italie et en Terre sainte, avait, au cours de sa jeunesse, fréquenté l'académie de Bologne, où il avait suivi les cours du maître d'armes Cavalcabo, dont il allait traduire le traité d'escrime. C'était une même formation militaire et mondaine que reçut Brantôme à Naples, en 1558, c'était la même que recherchait, une génération plus tard, un jeune gentilhomme qui laissa de son expérience italienne un très riche et très détaillé récit de voyage ${ }^{24}$. Lui-même avait visité comme un lieu obligé l'académie de Pignatelli, à Naples, où se formaient les meilleurs cavaliers du royaume et où il avait retrouvé de nombreux gentilshommes français ${ }^{25}$. Naples, à cette époque, ne bénéficiait d'aucun prestige savant, à 
l'exception du cabinet des frères Della Porta. C'était en outre une ville espagnole, et qui ne pouvait laisser au Français que la nostalgie d'une présence passée et des campagnes de Charles VIII. Or Naples attirait les Français, et c'est là, plus qu'en tout autre lieu, qu'ils trouvaient ce qui correspondait à leur propre idéal aristocratique, à leur culture nobiliaire, équestre et militaire. Cet attrait, qui est nettement manifesté dans les récits de voyage, allait jusqu'à prendre les formes d'un « lieu » de la fiction. Dans un roman de l'époque en effet, le père du héros,

craignant que les Lettres ne lui ôtassent la gaillardise requise en un homme de sa qualité pour paraître dans le monde, il lui manda que, cessant ses études, il s'acheminât à Naples pour apprendre les exercices ${ }^{26}$.

Montaigne n'alla pas jusqu'à Naples, mais de passage à Padoue, il visita lui aussi l'académie, négligeant l'université. Son secrétaire laissa le témoignage de cette visite :

Nous y fûmes tout le lendemain et vismes les écoles d'escrime, du bal, de monter à cheval, où il y avait plus de cent gentilshommes français ; ce que Monsieur de Montaigne comptait à grande incommodité pour les jeunes hommes de nostre pays qui y vont, d'autant que cette société les accoustume aux mœurs et langage de leur nation et leur ôte le moyen d'acquérir des connaissances étrangères ${ }^{27}$.

Montaigne, en gentilhomme, était attentif à ce lieu de formation pour les élites nobiliaires. Si le voyage d'Italie n'avait offert qu'un complément à une formation de juriste ou même d'érudit, il n'aurait été que d'un intérêt secondaire à ses yeux. Mais la critique que rapporte le secrétaire, et qui est le prolongement d'un passage fameux des Essais, témoigne aussi de la méprise que fait un homme plus âgé et plus mûr à propos du but que visaient les jeunes Français en venant en Italie. Ils ne cherchaient pas à se rendre italiens, à « rapporter principalement les humeurs de ces nations et leurs façons pour frotter et limer [leur] cervelle contre celle d'autruy $»^{28}$. À Padoue, ces jeunes gens restaient entre eux, et recevant un enseignement dispensé par des maîtres italiens, ils cherchaient à confirmer les qualités d'allure et de goûts qui distinguaient la noblesse française et lui donnaient son lustre propre. Leur formation était italienne, certes, par l'apprentissage d'arts et de techniques dans lesquelles les maîtres italiens affirmaient encore leur excellence (la danse, les armes, l'équitation), mais mis au service de l'idéal du gentilhomme français, au moment où, du fait des guerres civiles, aucune institution française n'en assumait l'enseignement. Montaigne expliquait par cette «malaisance » et aussi par le goût de la nouveauté l'élan qui poussait ses compatriotes vers l'Italie : «il ne se voit guères de Romain en l'escole de l'escrime à Rome, qui est pleine de Français ${ }^{29}$. Pierre de 
Lancre, qui avait été reçu docteur en droit à Turin en 1579, transcrivant les souvenirs de son propre séjour transalpin, formulait une idée semblable, à moins qu'il ne reprît cette idée aux Essais, mais sans témoigner d'ironie. Selon lui, la fréquentation des salles d'armes et des manèges, à Padoue, à Rome ou à Naples, restait le choix distinctif des Français :

Rome entre toutes les villes d'Italie est le vray rendez-vous des François. L'Alement, l'Espagnol, l'Anglois ny l'Italien mesme ne pratiquent guères les salles et escholes des exercices ${ }^{30}$.

Le voyage des jeunes Français, à la fin du XVI ${ }^{e}$ siècle, en dépit d'une analogie superficielle, était très différent dans ses modes et ses fins de ce qu' on appellera plus tard le Grand Tour ou le Kavaliertour des jeunes nobles de l'Europe septentrionale ${ }^{31}$. Il n'était pas entièrement organisé suivant un itinéraire touristique codifié, même s'il avait ses lieux communs visuels et narratifs, il ne privilégiait pas encore la seule expérience artistique dans une Italie qui n'était pas encore considérée comme le conservatoire des arts visuels. La noblesse française allait en Italie pour voir du pays, assurément, mais surtout pour se former, s'y retrouver, s'y faire connaître, dans la nostalgie des exploits militaires de ses ancêtres. Ce voyage, qui durait généralement moins d'une année, permettait au jeune homme ainsi formé d'entrer dans des réseaux et des clientèles, de faire ses premiers services, d'accéder à ses premiers emplois. En 1581, Arnaud du Ferrier, l'ambassadeur du roi à Venise, suggérait d'augmenter la suite de l'ambassadeur envoyé à Constantinople « du grand nombre de gentilshommes François qui sont aujourd'huy en Italie pour apprendre tous exercices ».

\section{La critique du voyage d'Italie}

À la fin du siècle, cette formation aristocratique, à l'instar de la formation universitaire, connaissait une crise. Le fils de Du Plessis-Mornay séjourna à Padoue en 1595, comme l'avait fait son père une génération plus tôt. Il constatait que les Français y étaient moins nombreux. Il attribuait cette désertion à des raisons religieuses ${ }^{32}$. Mais tous ses compatriotes n'étaient pas protestants ; la raison était plus profonde, et la désaffection, plus générale.

Le voyage d'Italie connut une critique diffuse, exprimée surtout en termes d'efficacité et d'utilité, en même temps qu'elle s'inscrivait dans une polémique anti-italienne plus générale. Cette critique, en apparence limitée et qui ne touchait qu'incidemment aux échanges savants, mettait en question l'expérience italienne et sa place dans la formation de la noblesse, elle insistait sur la différence radicale entre deux modèles, celui du courtisan 
italien et celui du gentilhomme français. Au moment où s'achevaient les guerres civiles et où les élites françaises se vouaient entièrement à la reconstruction du royaume, la formation de la noblesse et la répression d'une violence qu'elle avait jusqu'alors pu exprimer impunément furent au centre des préoccupations. Les plus attentifs aux nécessités d'une nouvelle «institution » des enfants pesaient les avantages et les inconvénients d'une expérience de l'Italie qui jusqu'alors avait si bien contribué à la culture et au prestige des élites françaises. En insistant, parfois de façon caricaturale sur les dangers auxquels les jeunes gens étaient exposés, certains hommes de lettres mettaient en évidence ce qu'ils présentaient comme des inconvénients pédagogiques de ce voyage et des risques économiques qu'il impliquait.

François de La Noue formula le problème dans les termes les plus clairs. À son époque, les jeunes gens avaient le choix entre l'Italie et l'Allemagne, selon leur appartenance religieuse. Cette formation qu'ils allaient chercher à l'étranger était fondée sur une illusion, qui faisait croire que « les drogues d'autrui sont meilleures que celles de leur pays ». L'Allemagne rendait les jeunes gens grossiers, sans politesse, inaptes à la vie de cour ; l'Italie les rendait plus civils, mais elle les exposait au risque de l'irréligion, à l'impiété, à la débauche, au luxe :

Quant au voyage d'Italie, plus y en a qui les font, principalement pour s'instituer en beaucoup d'exercices honnêtes qui y abondent. Mais parmi ces roses, on rencontre beaucoup d'espines pource qu'y ayant mille appasts de volupté, comme semés dans les plus belles villes, la jeunesse qui est désireuse de nouveauté et ardente dans ses affections, ne se peut retenir qu'elle n'aille gouster voire se saouler de ces très-douces poisons, et puis par la continuation s'engendre de très sales habitudes ${ }^{33}$.

Fort admiratif devant tout ce que l'Italie savait offrir dans les lettres et les arts, La Noue déconseillait pourtant d'y envoyer des adolescents encore trop peu formés. Seuls des jeunes gens bien préparés et bien encadrés pouvaient tirer profit d'un séjour dans la péninsule, et de façon provisoire seulement, en attendant que des institutions françaises se fussent substituées aux académies de Padoue ou de Naples. La Noue conseillait un séjour court, afin de limiter les risques de maladie et de duel, et surtout afin de restreindre la dépense, qui faisait de ce voyage un investissement trop lourd pour les ressources de la noblesse, un véritable gaspillage au moment où tout devait être utilisé pour la restauration du royaume ${ }^{34}$.

Cette institution que La Noue appelait de ses vœux, fut fondée quelques années plus tard. En 1599, dans son Académie ou institution de la noblesse françoise, qu'il dédiait à Henri IV, Alexandre de Pontaymery, un protestant qui avait lui-même passé deux années en Italie, reprenait tout un ensemble 
de lieux communs qu'il avait trouvés chez Henri Estienne ${ }^{35}$. Il voulait montrer quels étaient les vices de l'Italie et les dangers concrets du voyage, qui menaçaient des jeunes gens dont il dénonçait aussi les défauts de caractère et l'éducation imparfaite. Le jeune gentilhomme français, curieux de tout, parcourait l'Italie tel « un jeune cheval esgaré », dans un voyage de pur agrément, oubliant trop souvent « le chemin des exercices » qu'il devait suivre, et ne rapportait en fin de compte que « la seule ombre de la civilité ». Les promenades curieuses étaient un luxe bien vain, et l'attention portée aux antiquités se justifiait d'autant moins qu'elle ne servait à rien, selon Pontaymery, dans la conception d'une honnêteté qui s'édifiait précisément contre la tradition de l'humanisme érudit. La noblesse devait acquérir sa compétence militaire et administrative, tournée vers l'intérêt public plus qu'elle ne devait se consacrer au culte des Muses. Il proposait une solution purement française, qui devait permettre d'éduquer les jeunes gens dès le plus jeune âge, et qui avait surtout le mérite d'éviter « la despence incroyable $\gg$ du voyage :

Nous pouvons maintenant oublier ce chemin et prendre les erres de l'Académie du sieur de Pluvinel, qui nous fait voir en gros tous les exercices que l'Italie monstre en détail.

Pontaymery se livrait à l'éloge quasi publicitaire d'une académie, celle d'Antoine de Pluvinel, à laquelle le roi lui-même avait accordé son appui en lui confiant la formation du jeune duc de Vendôme ${ }^{36}$. Son ouvrage constituait son programme. C'est précisément autour de Pluvinel, le fondateur de l'école équestre française, que se développa cette polémique, dans les réflexions et les ouvrages de ses amis et collaborateurs Thomas Pelletier et Benoît Prévost. Les « exercices » italiens, enseignés par des maîtres qui possédaient les plus profonds secrets de leur art, avaient montré leur insuffisance. Les disciples des cavaliers italiens étaient revenus d'Italie déçus par un enseignement trop formel, dont ils critiquaient les « petits secrets », ceux en particulier d'une équitation de manège, inutile et inadaptée aux conditions de la guerre.

D'un point de vue plus général, le modèle italien de civilité, jadis capable de former le courtisan accompli de Castiglione, ne valait pas pour le gentilhomme français :

Ce n'est donc point un Italien qu'on a à nourrir. C'est un gentilhomme François qu'on désire avoir, les mœurs, la façon, la grâce vrayement à la Françoise et non à l'Estrangère. Ce sera donc en la seule France qu'il apprendra à estre à cheval, à courre la bague, à danser, à s'habiller à nostre mode sans estre jugé à son retour plus Italien que François, espargnant en cela le temps et la despense ${ }^{37}$. 
La richesse et les agréments que pouvaient offrir l'Italie ne lui étaient pas déniés. La critique du voyage ne portait pas sur une pratique à laquelle, par habitude et par goût les Français continuaient d'être attachés ; elle portait sur une des fins traditionnelles de ce voyage, remettant en question sa pertinence dans la seule formation des jeunes gens. On pouvait en somme continuer d'aller en Italie pour « voir », mais non plus pour apprendre. Dès le début du XVII ${ }^{\mathrm{e}}$ siècle, l'Italie avait cessé d'être considérée comme un lieu de formation et d'éducation aristocratique. Elle devenait en revanche un lieu de loisir pour des hommes mûrs, qui, n'ayant rien à apprendre, pouvaient la visiter sans risque. Le voyage d'Italie pouvait se vivre pleinement dans le temps de l'otium. D'abord temps privilégié favorables aux études, cet otium se transformait en loisir, délicieux mais moins urgent, et l'Italie devenait pour trois siècles le lieu de ce loisir.

\section{Université de Reims}

\section{Notes}

1. N. Bénard, Voyage de Hierusalem et autres lieux (Paris, D. Moreau, 1621), p. 589.

2. Voir à ce propos, J. Thuillier, " "Il se rendit en Italie"... Notes sur le voyage à Rome des artistes français au XVII ${ }^{\mathrm{e}}$ siècle », dans Études offertes à André Chastel (Paris, Flammarion ; Rome, Edizioni dell'Elefante, 1987), p. 321-36.

3. Lettre citée par J. Boucher, «Un grand aumônier engagé : Jacques Amyot et la cour de Henri III », dans Fortunes de Jacques Amyot, actes du colloque international, Melun, 18-20 avril 1985, éd. Michel Balard (Paris, Nizet, 1986), p. 148-49.

4. A. de Laval, Les Desseins des professions nobles et publiques (Paris, Abel L'Angelier, 1605), fol. 31.

5. Voir É. Picot, «Les Français dans les universités italiennes », Bulletin italien 17 (1917) : 172-84 et 18 (1918) : 28-36, repris dans Les Italiens en France au XVIe siècle (Bordeaux, Gounouilhou, 1918), rééd. N. Ordine (Rome, Vecchiarelli, 1995), p. 255-98. Un bon panorama de ces universités est donné par P. F. Grendler, The Universities of the Italian Renaissance (Baltimore, Johns Hopkins University Press, 2002).

6. Voir É. Picot, «Les professeurs et les étudiants de langue française à l'université de Pavie au XV ${ }^{\mathrm{e}}$ et au XVI ${ }^{\mathrm{e}}$ siècles », Bulletin philologique et historique (1915) : 8-90 et (1917) : $71-73$.

7. É. Picot, « Histoire de l'Université de Ferrare », Journal des Savants (1902) : 80-102 et 141-58.

8. N. Audebert, Voyage d'Italie, éd. A. Olivero (Rome, Lucarini, 1981).

9. Voir B. Brugi, «Gli antichi scolari di Francia allo studio di Padova », dans Mélanges offerts à M. Émile Picot, 2 vol. (Paris, H. Champion, 1913), 1 : 535-55.

10. Voir en particulier H. Busson, Les Sources et le développement du rationalisme dans la littérature française de la Renaissance (1531-1601), « Bibliothèque de la Société d'Histoire ecclésiastique de la France » (Paris, Letouzey et Ane, 1922). 
11. On pourra comparer la situation et les habitudes des étudiants français à celles de leurs condisciples anglais ; voir K. R. Bartlett, The English in Italy (1525-1558) (Genève, Slatkine, 1991), p. 29-58.

12. P. E. Virey, Vers itinéraires, éd. A. Bettoni (Paris, S.T.F.M., 1999).

13. P. Gassendi, Viri illustris Nicolai Claudii Fabricii de Peiresc Vita [1641] (La Haye, Vlacq, 1655), p. 11.

14. Peiresc, Lettres, éd. Ph. Tamisey de Larroque, vol. 6, « Collection de documents inédits sur l'histoire de France » (Paris, Imprimerie Nationale, 1896), p. 6.

15. Voir sur ce point M. Fumaroli, «L'Inspiration du poète » de Poussin. Essai sur l'allégorie du Parnasse (Paris, Réunion des Musées nationaux, 1989).

16. J.-A. de Thou, Mémoires de sa vie (Rotterdam, Leers, 1711), p. 15-34.

17. Sur ce point, voir notre étude, «Larivey traducteur de l'Institution morale de Piccolomini », dans Pierre de Larivey (1541-1619), éd. Y. Bellenger (Paris, Klincksieck, 1993), p. 73-82.

18. La Correspondance entre deux humanistes, G. V. Pinelli et C. Dupuy, a été éditée par A. M. Raugei (Florence, L. Olschki, 2001).

19. A. Boniel, La Vie de messire Claude Expilly (Grenoble, Ph. Charvys, 1640), p. 15. Expilly séjourna à Padoue entre 1580 et 1582.

20. Sur la suite des rencontres savantes en Italie, voir P. Dibon et Fr. Waquet, Johannes Fredericus Gronovius pèlerin de la République des Lettres. Recherches sur le voyage savant au XVIIe siècle (Genève, Droz, 1984), p. 1-36.

21. A. Desjardins, Négociations diplomatiques entre la France et la Toscane, documents recueillis pas G. Canestrini, «Collection de documents inédits sur l'histoire de France » (Paris, Imprimerie Nationale, 1859-1886), $4: 534$ et 680.

22. T. Tasso, Delle rime et prose (Venise, G. Vasalini, 1586), p. 128 et 136.

23. Ch. de Villeroy, Memoire de tout mon Voege faict en Italie lan 1583 avec les choses remercables que j'ay veues, Ms. BNF fr. 14660, fol. 25.

24. Discours viatiques de Paris à Rome et de Rome à Naples (1588-1589), éd. L. Monga (Genève, Slatkine, 1983), p. 108.

25. Sur la formation italienne des cavaliers français, voir notre étude «Montaigne, le style (du) cavalier et ses modèles italiens », Nouvelle revue du seizième siècle 17.2 (1999) : 253-68.

26. Des Ecuteaux, Les chastes Amours de Clarimond et Antonide (Rouen, Jean Osmont, 1602), p. 5.

27. Montaigne, Voyage, éd. F. Garavini (Paris, Gallimard, 1983), p. 160.

28. Montaigne, Les Essais, éd. Pierre Villey, nouvelle éd. (Paris, Presses Universitaires de France, 1965), I, 26, p. 153.

29. Ibid., II, 15, p. 613.

30. P. de Lancre, Tableau de l'inconstance et instabilité de toutes choses (Paris, Veuve L'Angelier, 1610), fol. $438^{\mathrm{v}}$.

31. Voir E. Chaney, The Grand Tour and The Great Rebellion: Richard Lassels and «The Voyage of Italy » in the Seventeenth Century (Genève, Slatkine, 1985).

32. Mme de Mornay, Mémoires, éd. Mme de Witt, vol. 1 (Paris, Veuve J. Renouard pour la Société de l'Histoire de France, 1868), p. 28-35 ; sur ce point, voir A. Stella, « Attegiamenti 
eterodossi di studenti francesi nell'università di Padova fra Cinque e Seicento », dans Échanges religieux entre la France et l'Italie, éd. M. Maccarrone et A. Vauchez (Genève, Slatkine, 1987), p. 287-300.

33. Fr. de La Noue, Discours politiques et militaires [Bâle, 1587] (La Rochelle, Villepoux, 1590), p. 162-71.

34. Sur ces aspects économiques, voir E. Giddey, «Les conditions matérielles et spirituelles du voyage d'Italie à la fin du XVI ${ }^{\mathrm{e}}$ siècle et au début du XVII ${ }^{\mathrm{e}}$ siècle », Études de lettres 4 (1960) : 173-95.

35. A. de Pontaymery, L'Académie ou institution de la noblesse françoise (Paris, J. Richer, 1595, rééditée dans Les CEuvres, ibid., 1599), fol. 2-7 et 40-58. Cette critique a été analysée par R. Bady, L'Homme et son institution (Paris, Les Belles Lettres, 1964), p. 387-98. Sur Estienne, voir L. Sozzi, « La polémique anti-italienne dans l'œuvre narrative de Henri Estienne », dans Henri Estienne, « Cahiers V.-L. Saulnier » (Paris, Presses de l'École Normale Supérieure, 1988), p. 97-111.

36. Voir H. Nelson, « Antoine de Pluvinel Classical Horseman and Humanist », French Review 58 (1985) : 514-23.

37. Th. Pelletier, La Nourriture de la Noblesse (Paris, Veuve Patisson, 1604), fol. 96. Ce texte est précédé de vers de Benoît Prévost. Ce dernier publia un manuel d'art équestre, Le Cavesson françois (Poitiers, C. Pignon et C. Courtois, 1610), dans la préface duquel il critiquait à nouveau le voyage de formation équestre en Italie. 